\title{
Cut and paste the genome: Genome editing for research and therapy
}

\author{
Seahyoung Lee ${ }^{\mathrm{a}, \mathrm{b}}$, Chang Yeon Lee ${ }^{\mathrm{c}}$, Jiyun Lee ${ }^{\mathrm{d}}$, Hyang-Hee Seo ${ }^{\mathrm{d}}$ and Ki-Chul Hwang, ${ }^{\mathrm{a}, \mathrm{b}, *}$ \\ ${ }^{a}$ Institute for Bio-Medical Convergence, College of Medicine, Catholic Kwandong University, \\ Gangneung-si, Gangwon-do, Korea \\ ${ }^{\mathrm{b}}$ Catholic Kwandong University International St. Mary's Hospital, Incheon Metropolitan City, Korea \\ ${ }^{\mathrm{c}}$ Department of Integrated Omics for Biomedical Sciences, Yonsei University, Seoul, Korea \\ ${ }^{\mathrm{d}}$ Brain Korea 21 PLUS Project for Medical Science, Yonsei University, Seoul, Korea
}

\begin{abstract}
Genome engineering, or alternatively called 'genome editing,' has been one of the rapidly growing fields of biotechnology for the last few decades. Scientists are now making targeted modifications of genome in any organism of choice with improved precision. In this mini review, we provide basic, fundamental theory and mechanisms of the well-known genome editing technologies such as zinc-finger nucleases (ZFNs), transcription activator-like effector nucleases (TALENs) and clustered regulatory interspaced short palindromic repeats (CRISPR)/Cas9 system. We also discuss its role in the field of genetic research and highlight its therapeutic potential as an indispensable tool for the development of personalized medicine to come in near future.
\end{abstract}

\section{Introduction}

For the last few decades, the biotechnology to decipher individual human genome such as wholegenome sequencing (WGS, [1]) and whole-exome sequencing (WES, [2]) advanced greatly, lowering barriers to decoding the information embedded in human genome. Such technological advances are anticipated to revolutionize our understanding of clinical genetics and to deliver personalized medicine in near future. Nevertheless, the present challenge lies in converting this tremendous amount of data (i.e., billions of nucleotides that comprise DNA of an individual) into clinically relevant information, such as how genotype affects phenotype. To transform the data into functionally relevant knowledge, technologies such as targeted gene inactivation by homologous recombination [3] or targeted gene knockdown by RNAi [4] have been utilized as means of providing empirical information to elucidate the function of genes of interest. However, the former has limitations such as extremely low efficiency of correct insertion into the target chromosome ( 1 in $10^{6} \sim 1$ in $10^{9}$ ) [5] and potential adverse mutagenesis [6], while the latter has limitations including incomplete and temporary knockdown of target gene and undesired off-target effects [7]. More recently, genome engineering technologies, commonly referred to as 'genome editing,' have been emerged, enabling scientists to make targeted modifications to the genome in practically any organism of choice with improved precision [7a]. This technology utilizes engineered nucleases which

\footnotetext{
*Corresponding author: Ki-Chul Hwang. E-mail: kchwang@cku.ac.kr.
} 
are complex of sequence-specific DNA binding domains and nonspecific DNA cleavage modules [8]. In this brief review, we aim to provide basic information on this genome editing technologies and discuss the applications and the therapeutic potential of these technologies, as well as future prospects.

\section{Principle mechanisms of genome editing technologies}

Genome editing technology involves customized programmable DNA-binding nucleases such as zincfinger nucleases (ZFNs) [8], transcription activator-like effector nucleases (TALENs) [9], and clustered regularly interspaced short palindromic repeats (CRISPR)/Cas9 (CRISPR-associated 9) nucleases [10]. Although each nuclease has unique mode of action, in principle, these nucleases recognize, bind to, and cleave chromosomal DNA to create site-specific DNA double strand breaks (DSBs). Subsequently, these DSBs trigger endogenous DNA repair systems, such as homology directed repair (HDR) or error-prone non-homologous end joining (NHEJ), resulting in targeted genome modification (Fig. 1) [11].

\section{Programmable DNA-binding nucleases}

\subsection{Zinc-finger nucleases (ZFNs)}

First discovered in transcription factor from Xenopus laevis [12], Cys2-His2 zinc-fingers are the most common sequence-specific DNA-binding motifs found in all eukaryotic organisms [13]. Each zinc-finger

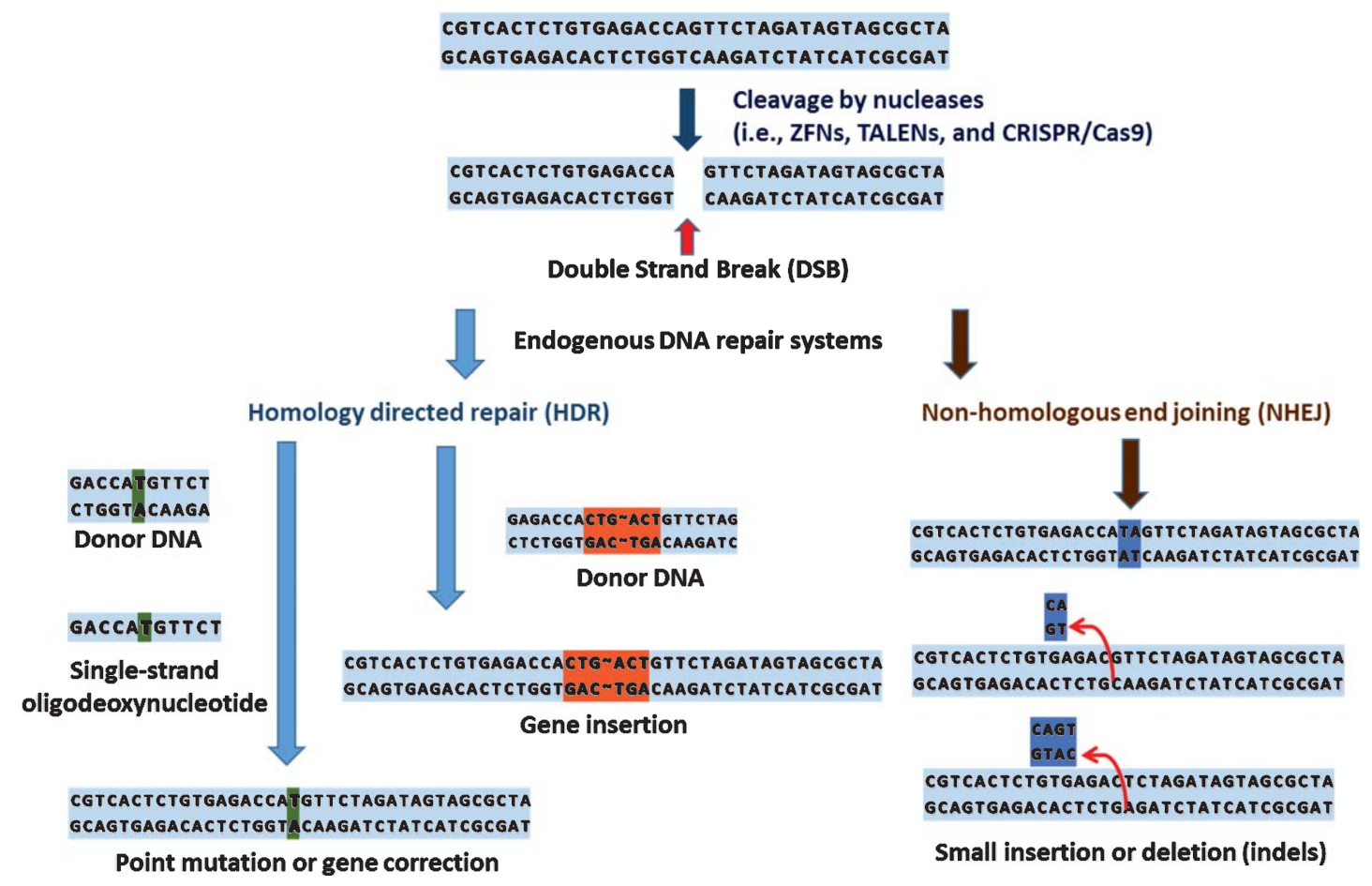

Fig. 1. Schematics of genome editing process. 
motif consists of about 30 amino acids in a $\beta \beta \alpha$ configuration, and two cysteines and two histidines coordinate a single zinc atom in individual zinc-finger [14]. Amino acids in positions $-1,3$, and 6 on the $\alpha$-helix contact 3 base pairs in the major groove of DNA [15]. Thus, theoretically, a combination of 6 separate zinc-fingers that each recognizes a 3 base pair DNA sequence can consequently recognize a specific DNA sequence of 18 base pairs, which is long enough sequence to specify a unique site in the human genome. The construction of such unnatural, synthetic zinc-fingers that recognized 18 base pairs of DNA sequence made application of zinc-finger proteins for recognizing specific DNA sequence possible [16]. However, it was the combination of zinc-finger proteins with cleavage domain of endonuclease that made possible to realize the concept of targeted genome engineering. Isolated from Flavobacterium okeanokoites, the type II restriction endonuclease Fok I recognizes the DNA sequence of 5'-GGATG-3' and cleaves 9th and 13th base pairs from the recognition site, regardless of the sequence at the cleaved site [17]. When the cleavage domain of Fok I endonuclease was combined with zinc-finger protein to generate zinc-finger-Fok I fusion proteins, these hybrid fusion proteins (ZFNs) were able to cut DNA at the predetermined sequences [18].

Since Fok I cleavage domain must dimerize to catalyze DNA cleavage, during ZFN-mediated site specific DNA cleavage, ZFN target sites consisted of two zinc-finger binding sites separated by $5 \sim 7$ base pairs of spacer sequence are occupied by two ZFNs on each strand and the cleavage domain of each ZFN dimerize on the spacer sequence and cleaves it (Fig. 2). Currently there are 3 large sets of constructed ZF proteins (ZFPs) from independent groups, namely The Scripps Research Institute [19-21], Sangamo Biosciences [22], and ToolGen [23], are available. Recently published articles on the use of ZFNs are listed in Table 1 . The contents of Table 1 are not based on scientific significance by any means. It is just examples of recent research trend involving use of ZFNs. As demonstrated in the Table below, researches on ZFNs are still active, and most of the cases the ZFNs are utilized as a tool for studying function of

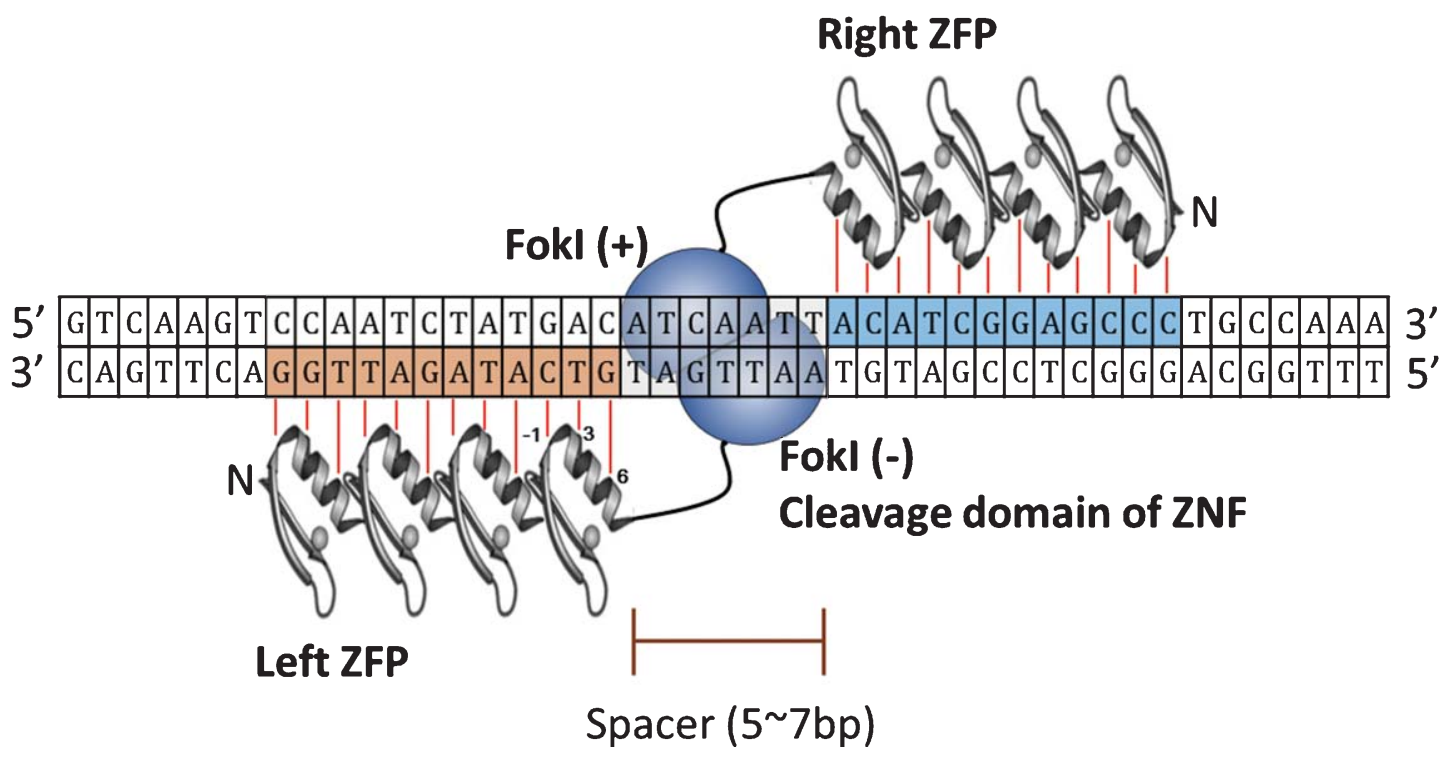

Fig. 2. ZFN dimerize on the spacer sequence via sequence-specific interaction between ZFP and DNA. Amino acids in positions $-1,3$, and 6 on the $\alpha$-helix recognizes and contact 3 base pairs in the major groove of DNA. FokI cleavage domains from each ZFN dimerize over the spacer sequence to facilitate DBS. 
Table 1

Selected recent examples of ZFN-mediated genome editing in various organisms including human cells

\begin{tabular}{llll}
\hline Gene modification & Organism & Genes & Refs \\
\hline Disruption & Mouse & tbc1d20, rab3gap1, rab3gap2, rab18 & {$[24]$} \\
& Sheep & mstn & {$[25]$} \\
& Human & MDR1, MRP2, BCRP & {$[26]$} \\
& Human & HPV E7 & {$[27]$} \\
& Humanized mouse & CCR5 & {$[28]$} \\
Addition & Cow & hLYZ & {$[29]$} \\
& Human & CCR5 & {$[30]$} \\
Correction & Human & AAVS1 & {$[31,32]$} \\
& Human & HBB & {$[33,34]$} \\
\hline
\end{tabular}

Few selected, recent articles searched through Pubmed. The literature search was to overview the research trend regarding ZFN-mediated genome editing.

A

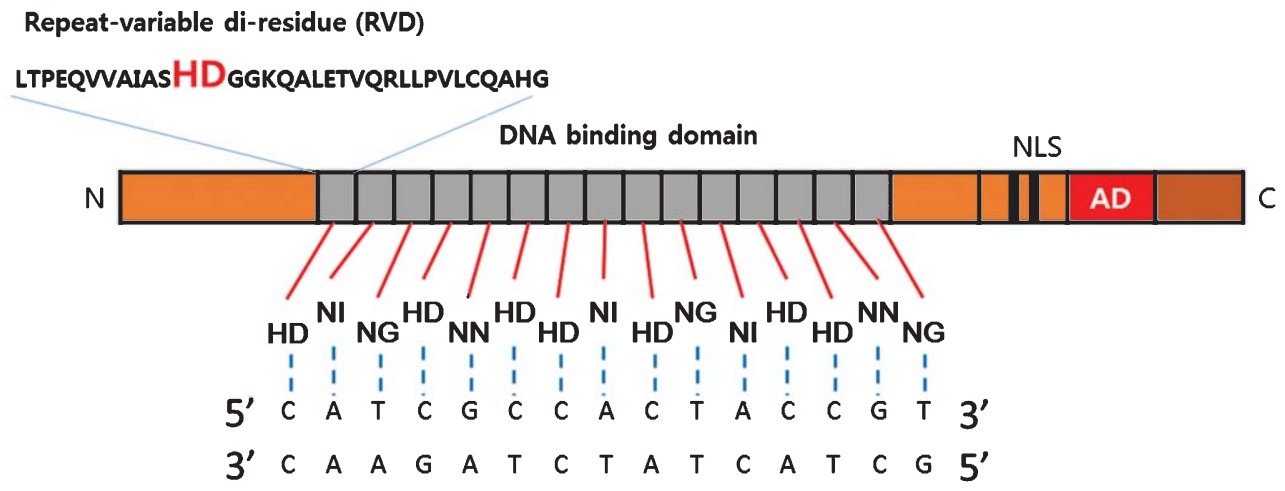

B

LTPEQVVAIASHDGgKQALETVQRLLPVLCQAHG

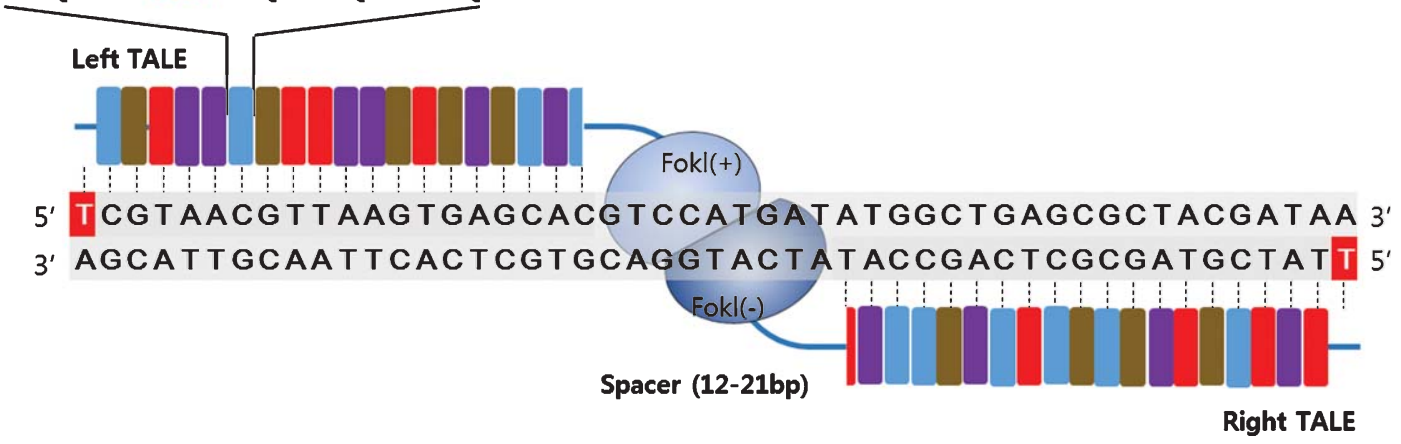

Fig. 3. Structures of TALE and TALEN. A. Schematic presentation of TALE (TAL effector). Each TALE repeat contains 33-35 amino acids, and each RVD (amino acids at positions 12 and 13) recognizes a corresponding single base pair. RVD within a consensus repeat sequence is indicated in red. NLS: nuclear localization signal, AD: transcriptional activation domain. B. TALEN pair binds to specific DNA sequence recognized by TALEs. FokI cleavage domains from each TALE dimerize over the spacer sequence. In most cases, naturally occurring TALEs are preceded by $\mathrm{T}$ at the 5 '-end [39] as indicated by red box. 
certain genes by inducing gene disruptions. However, there are also studies to correct mutations in human cells, implicating the therapeutic potential of genome editing using ZFNs.

\subsection{Transcription activator-like effector nucleases (TALENs)}

TAL (transcription activator-like) proteins were first identified as secreted proteins from bacterial plant pathogen Xanthomonas, and the named based on observation that they activated transcription of endogenous pathogenic genes in plants $[35,36]$. Once they enter the nucleus of host cells, they bind to effector-specific sequences on the promoters of host genes to initiate transcription [37]. TALENs are composed of DNA-binding domain (DBD), N-terminal, C-terminal, and FokI endonuclease domain. The DBD of TALEN is composed of 'repeats' that itself composed of 33 35 amino acids [38].

Among these amino acids, the amino acid residues at position 12 and 13 of each repeat decide which nucleotide of DNA they bind to (i.e., Asparagine-Isoleucine (NI) $\longrightarrow$ Adenine (A), AsparagineGlycine $(\mathrm{NG}) \longrightarrow$ Thymine $(\mathrm{T})$, Aspargine-Asparagine $(\mathrm{NN}) \longrightarrow$ Guanine $(\mathrm{G})$, Histidine-Aspartic Acid (HD) $\longrightarrow$ Cytosine (C)) so they are named as 'repeat-variable di-residue (RVD) [39]. It is this one RVD to one nucleotide match between the RVD of TALENs and chromosomal DNA that underpins target sequence specificity of TALENs (Fig. 3A). Furthermore, this sequence specific interaction and simplicity of its coding system made generation of customized TALE domains that target DNA sequence of interest possible $[40,41]$.

The TALE domains can be assembled in predetermined combinations and linked to the Fok I nuclease to drive sequence-specific DSB of chromosomal DNA [42]. TALENs, in pairs, bind to opposing target sequences so that the linked Fok I nucleases come in contact with the spacer from opposite sides. The

Table 2

Selected recent examples of TALEN-mediated genome editing in various organisms including human cells

\begin{tabular}{|c|c|c|c|}
\hline Gene modification & Organism & Genes & Refs \\
\hline \multirow[t]{10}{*}{ Disruption } & Mouse & c-kit, PU.1 & [43] \\
\hline & Zebrafish & nptx2a & {$[44]$} \\
\hline & $X$. tropicalis & $\mathrm{TR} \alpha$ & {$[45]$} \\
\hline & Human & Rap1 & [46] \\
\hline & Human & Gads & [47] \\
\hline & Zebrafish & rspo2 & [48] \\
\hline & Mouse & ace 2 & [49] \\
\hline & Zebrafish & fshb, lhb & {$[50]$} \\
\hline & $X$. tropicalis & dot11 & [51] \\
\hline & Human & $\operatorname{miR}-21$ & [52] \\
\hline Addition & Human & eGFP & [53] \\
\hline \multirow[t]{5}{*}{ Correction } & Human & DMD & {$[54,55]$} \\
\hline & Human & CCR5 & [56] \\
\hline & Human & HBB & [57-59] \\
\hline & Mouse & Crb1 & [60] \\
\hline & Human & XPC & {$[61]$} \\
\hline
\end{tabular}

Few selected, recent articles searched through Pubmed. The literature search was to overview the research trend regarding TALEN-mediated genome editing. 
Fok I nucleases from each TALEN forms Fok I dimer and it cleaves double-stranded DNA (Fig. 3B). As indicated in the Table 2, TALEN-mediated genome editing is also widely used to facilitate gene disruption, addition, and correction.

\subsection{Clustered regulatory interspaced short palindromic repeat (CRISPR)/Cas9}

The existence of CRISPR was first discovered by Nakata et al. in studying iap gene of E.coli [62]. What they found was 29 nucleotide-long repeats interspaced by 5 intervening 32 nucleotide-long, nonrepetitive sequences. The actual word CRISPR for describing such repeat sequences was first coned by Jansen in 2002 [63]. The CRISPR loci are composed of a set of CRISPR-associated (Cas) genes and a series of repeats (direct repeats, approximately $20-50$ base pairs) interspaced by unique, non-repetitive sequences (spacers).

These non-repetitive sequences correspond to the sequences of foreign genetic elements (protospacers) [64]. the protospacers are flanked by a short protospacer adjacent motif (PAM) that is either located on the 3' (type II CRISPR) or 5' (type I CRISPR) of foreign DNA [65]. Additionally, leader sequence, rich in A+T sequences, is known to serve as a promoter element for the CRISPR loci [66] (Fig. 4). CRISPR is an essential components of RNA-based adaptive immune systems of bacteria and archaea [67]. In response to foreign genetic element challenges, bacteria and archaea incorporate short fragments of foreign genetic elements into host genome as shown in Fig. 4. When CRISPR locus is transcribed, the long primary transcript is processed to produce a library of short CRISPR-derived RNAs (crRNAs) [68, 69]. Each crRNA has a complementary sequence to a previously encountered foreign genetic elements, and it mediates detection and subsequent destruction of foreign nucleic acids [70]. This unique immune system can be divided into 3 distinct CRISPR types (type I-III) based on gene conservation and locus organization [71]. Especially in the type II CRISPR/Cas system, crRNAs hybridize with trans-activating crRNAs (tracrRNAs) to facilitate RNA-guided sequence-specific DNA cleavage by Cas9 proteins. Cas9 utilizes RNA-DNA pairing to target foreign DNA, and Cas9-RNA complex-mediated DNA cleavage requires recognition of PAM where DNA strand separation and RNA-DNA hybrid formation occurs (Fig. 5) [72].

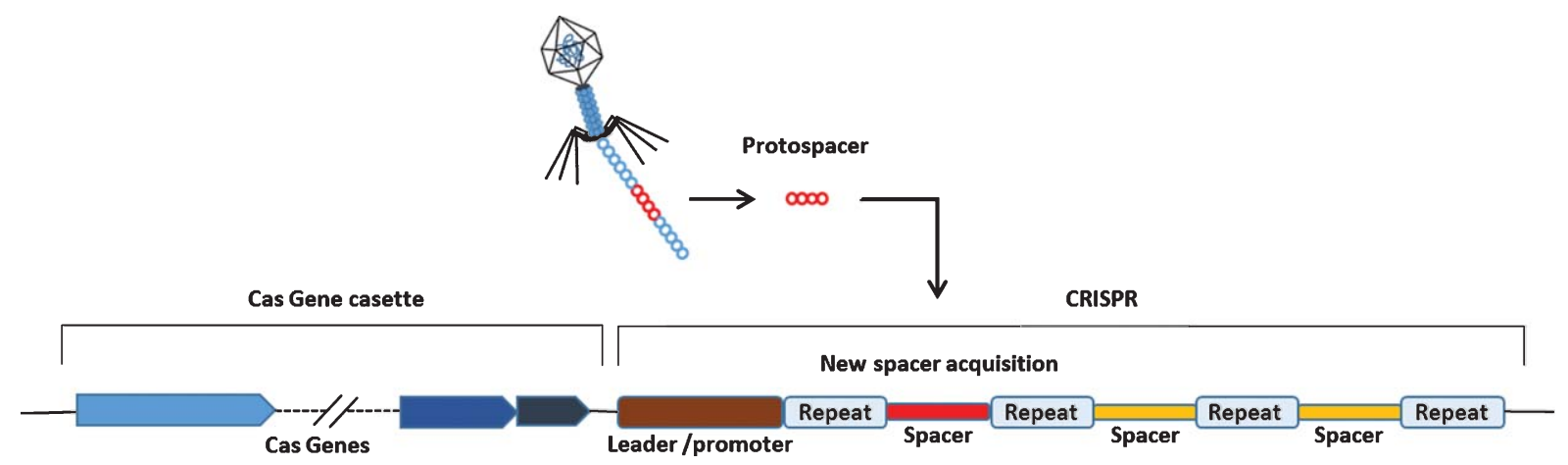

Fig. 4. Schmatics of a generalized CRISPR locus. Upon introduction of foreign genetic elements from bacteriophages or plasmids, Cas proteins obtain spacers from the exogenous protospacer sequences and they are incorporated into the CRISPR locus of host genome. 


\section{Biosynthesis and processing of crRNA}
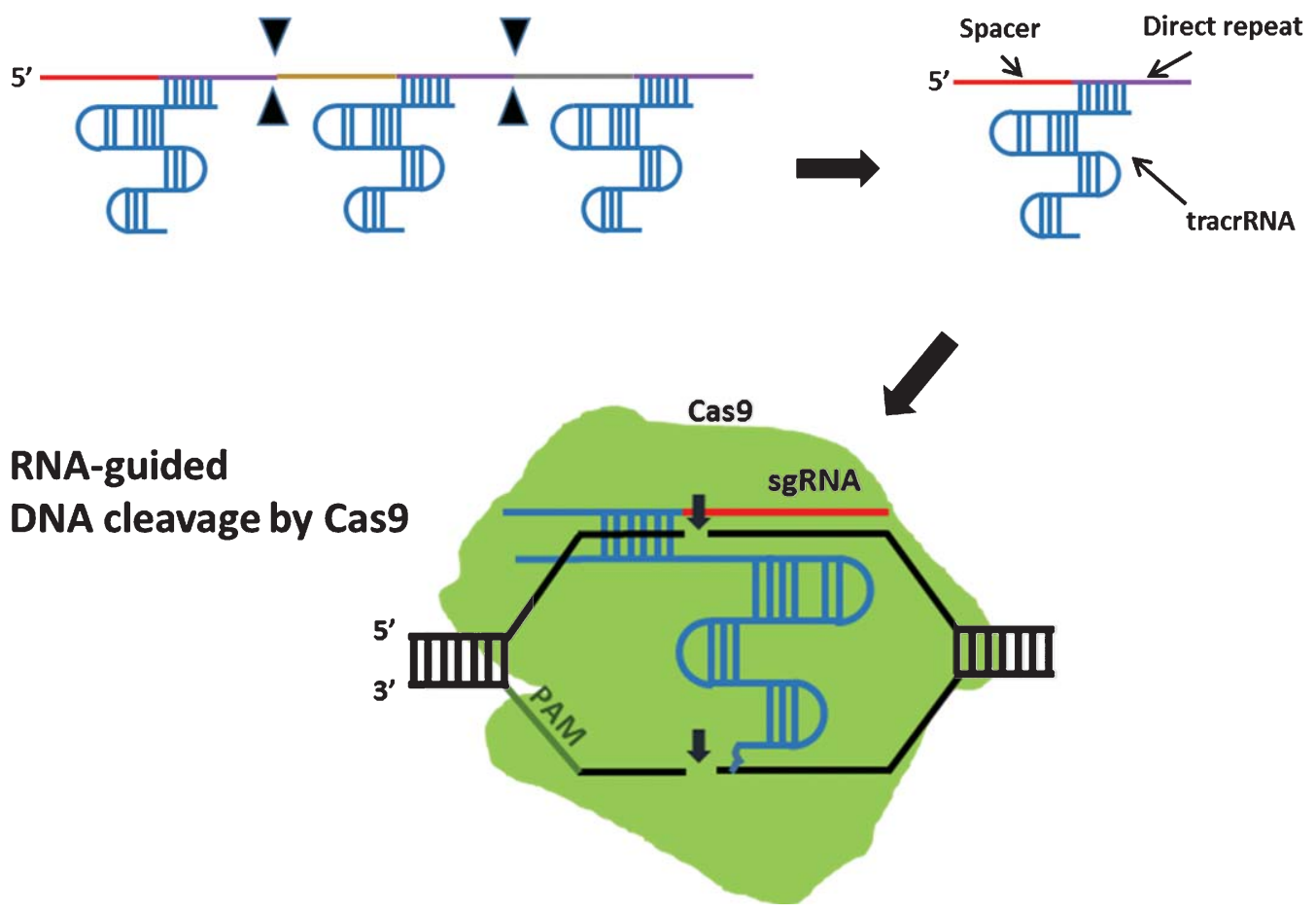

Fig. 5. DNA cleavage by type II CRISPR nuclease system. In type II CRISPR, crRNA-tracrRNA hybrids complex with Cas9 to facilitate sequence-specific DNA cleavage. Filled triangle indicates cleavage by Cas ribonucleases. Small arrows indicate DNA cleavage by Cas9. sgRNA: single guide RNA.

Table 3

Selected recent examples of CRISPR/Cas-mediated genome editing in various organisms including human cells

\begin{tabular}{lllr}
\hline Gene modification & Organism & Genes & Refs \\
\hline Disruption & Mouse & PrP & {$[73]$} \\
& Human & CCR5 & {$[74]$} \\
& human & PVALB, EMX1 & {$[75]$} \\
Addition & Human & AAVS1 & {$[76]$} \\
Correction & Mouse & Crygc & {$[77]$} \\
& Human & DMD & {$[54]$} \\
& Mouse & DMD & {$[78]$} \\
\hline
\end{tabular}

Few selected, recent articles searched through Pubmed. The literature search was to overview the research trend regarding CRISPR/Cas-mediated genome editing.

DNA sequence. In fact, reprogrammed RNA-guided nucleases have been demonstrated to be competent in facilitating gene disruption, addition, and correction in human cells and other model organisms (Table 3). 
Compared to ZFNs and TALENs, CRISPR/Cas9 system has higher efficiency and shortest target length, and it can also facilitate multiplex targeting [79]. Furthermore, Cas9 can be used for applications other than usual category of gene modifications (disruption, addition, and correction), such as, but not limited to, transcriptional control or DNA labeling $[80,81]$.

\section{Perspectives: The role of genome editing in achieving personalized medicine}

Although it is in its infancy, over the last few decades, genome editing has come of age and tremendous interest has been placed on its utility as a tool for basic/clinical genetics. Accumulating data also indicate its potential as a dexterous and powerful means of personalized medicine which is expected to provide individual patient with 'tailored' therapeutics near future. Promising results using these site-specific nucleases in therapeutic approaches against severe combined immune deficiency (SCID) [82], sickle cell disease [83], and hemophilia B [83], make the hopes of personalized medicine up ever more. For 'personalized medicine' to be realized, close cooperation of related biotechnologies and disciplines is required (Fig. 6).

For example, an ideal starting point of personalized medicine would be genomic information analysis of an individual patient. Biotechnologies such as WGS and WES would be a valuable tool for this task. From

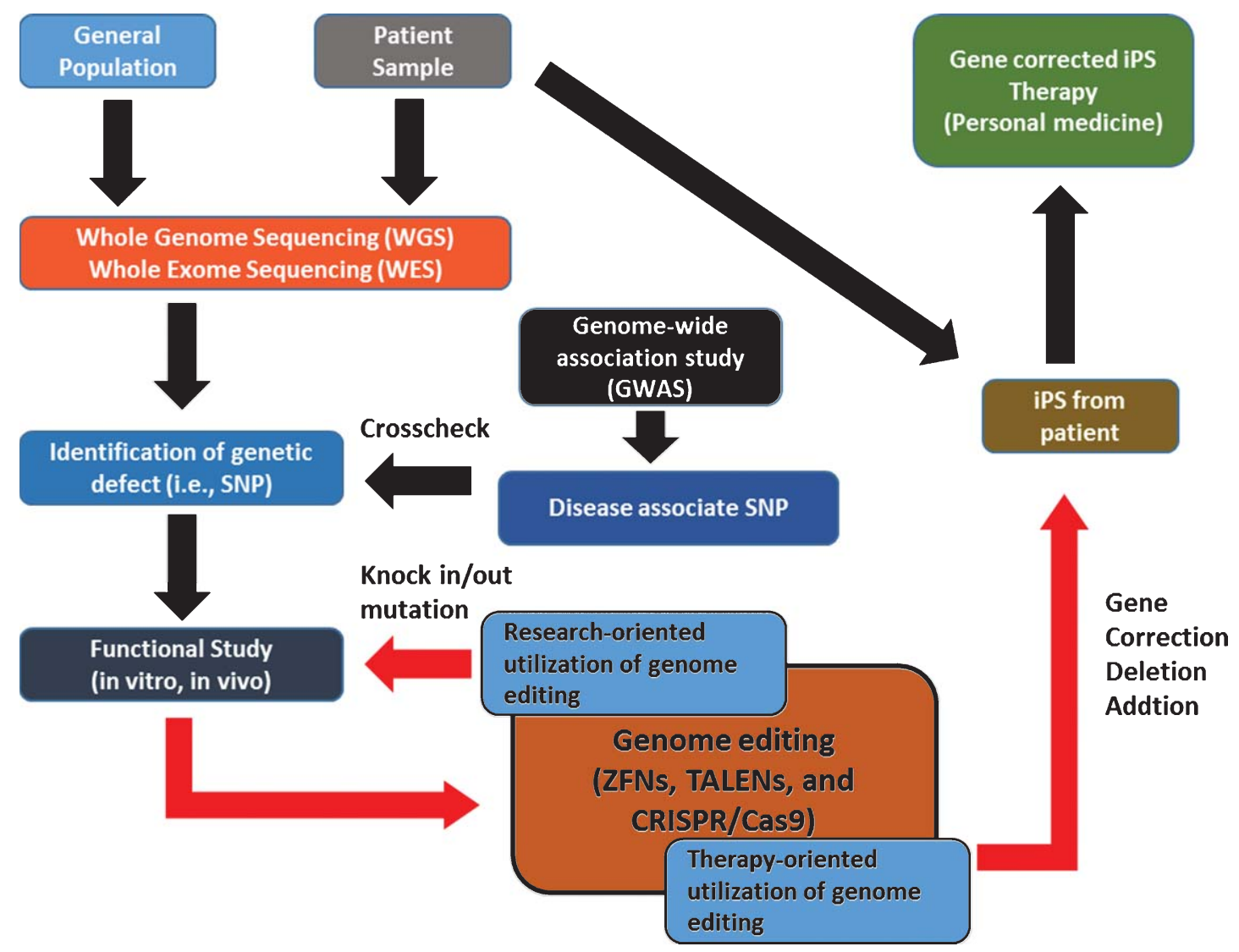

Fig. 6. Example of genome editing utilized in personalized medicine. 
the genomic analysis by sequencing can identify specific genetic defects of patients (i.e., single nucleotide polymorphism, SNP), and the relationship between specific SNP and phenotypic manifestation can be systemically examined both in vitro and in vivo. At this stage comes the genome editing technology. With the genome editing technology (i.e., gene knock in/out, point mutation, gene correction, and etc.) the functional consequences of a certain SNP can be determined in details (research-oriented utilization of genomic editing). Furthermore, genomic editing can serve as a part of therapeutic process that requires genomic modifications (i.e., gene correction of induced pluripotent stem cells (iPS) from specific patient). Thus, the genome editing technology can be both research-oriented and therapy-oriented applications. Although there are still some unsolved issues regarding detailed mechanisms of individual genome editing such as how foreign sequences are selected and incorporated into the CRISPR loci of host, it is undoubtful that these technology eventually revolutionizes the field of genetic research as well as our understanding of diseases and opens a new paradigm in fighting currently incurable diseases.

\section{Acknowledgments}

This study was supported by a Korea Science and Engineering Foundation grant funded by the Korean government (MEST) (2014030459) and a grant from the Korea Health 21 R\&D Project, Ministry of Health \& Welfare, Republic of Korea (A120478).

\section{Conflicts of interest}

The authors declare no conflict of interest.

\section{References}

[1] Ng PC, Kirkness EF. Whole genome sequencing. Methods in Molecular Biology 2010;628:215-26.

[2] Rabbani B, Tekin M, Mahdieh N. The promise of whole-exome sequencing in medical genetics. Journal of Human Genetics 2014;59:5-15.

[3] Capecchi MR. Gene targeting in mice: Functional analysis of the mammalian genome for the twenty-first century. Nature Reviews Genetics 2005;6:507-12.

[4] Mohr SE, Smith JA, Shamu CE, Neumuller RA, Perrimon N. RNAi screening comes of age: Improved techniques and complementary approaches. Nature Reviews Molecular Cell Biology 2014;15:591-600.

[5] Capecchi MR. Altering the genome by homologous recombination. Science 1989;244:1288-92.

[6] Vasquez KM, Marburger K, Intody Z, Wilson JH. Manipulating the mammalian genome by homologous recombination. Proceedings of the National Academy of Sciences of the United States of America 2001;98: 8403-10.

[7] Moore CB, Guthrie EH, Huang MT, Taxman DJ. Short hairpin RNA (shRNA): Design, delivery, and assessment of gene knockdown. Methods in Molecular Biology 2010;629:141-58.

[7a] Wang W, Xu X, Li Z, Lendlein A, Ma N. Genetic engineering of mesenchymal stem cells by non-viral gene delivery. Clin Hemorheol Microcirc 2014;58:19-48.

[8] Urnov FD, Rebar EJ, Holmes MC, Zhang HS, Gregory PD. Genome editing with engineered zinc finger nucleases. Nature Reviews Genetics 2010;11:636-46.

[9] Joung JK, Sander JD. TALENs: A widely applicable technology for targeted genome editing. Nature Reviews Molecular Cell Biology 2013;14:49-55.

[10] Maggio I, Holkers M, Liu J, Janssen JM, Chen X, Goncalves MA. Adenoviral vector delivery of RNA-guided CRISPR/Cas9 nuclease complexes induces targeted mutagenesis in a diverse array of human cells. Scientific Reports 2014;4:5105.

[11] Kim H, Kim JS. A guide to genome engineering with programmable nucleases. Nature Reviews Genetics 2014;15:321-34.

[12] Miller J, McLachlan AD, Klug A. Repetitive zinc-binding domains in the protein transcription factor IIIA from Xenopus oocytes. The EMBO Journal 1985;4:1609-14. 
[13] Pabo CO, Peisach E, Grant RA. Design and selection of novel Cys2His2 zinc finger proteins. Annual Review of Biochemistry 2001;70:313-40.

[14] Beerli RR, Barbas CF, 3rd. Engineering polydactyl zinc-finger transcription factors. Nature Biotechnology 2002;20:135-41.

[15] Pavletich NP, Pabo CO. Zinc finger-DNA recognition: Crystal structure of a Zif268-DNA complex at 2.1 A. Science 1991;252:809-17.

[16] Liu Q, Segal DJ, Ghiara JB, Barbas CF, 3rd. Design of polydactyl zinc-finger proteins for unique addressing within complex genomes. Proceedings of the National Academy of Sciences of the United States of America 1997;94:5525-30.

[17] Sugisaki H, Kanazawa S. New restriction endonucleases from Flavobacterium okeanokoites (FokI) and Micrococcus luteus (MluI). Gene 1981;16:73-8.

[18] Kim YG, Cha J, Chandrasegaran S. Hybrid restriction enzymes: Zinc finger fusions to Fok I cleavage domain. Proceedings of the National Academy of Sciences of the United States of America 1996;93:1156-60.

[19] Dreier B, et al. Development of zinc finger domains for recognition of the 5'-CNN-3' family DNA sequences and their use in the construction of artificial transcription factors. The Journal of Biological Chemistry 2005;280:35588-97.

[20] Dreier B, Beerli RR, Segal DJ, Flippin JD, Barbas CF, 3rd. Development of zinc finger domains for recognition of the 5'-ANN-3' family of DNA sequences and their use in the construction of artificial transcription factors. The Journal of Biological Chemistry 2001;276:29466-78.

[21] Segal DJ, Dreier B, Beerli RR, Barbas CF, 3rd. Toward controlling gene expression at will: Selection and design of zinc finger domains recognizing each of the 5'-GNN-3' DNA target sequences. Proceedings of the National Academy of Sciences of the United States of America 1999;96:2758-63.

[22] Liu Q, Xia Z, Zhong X, Case CC. Validated zinc finger protein designs for all 16 GNN DNA triplet targets. The Journal of Biological Chemistry 2002;277:3850-6.

[23] Bae KH, et al. Human zinc fingers as building blocks in the construction of artificial transcription factors. Nature Biotechnology 2003;21:275-80.

[24] Park A, Liegel RP, Ronchetti A, Ebert AD, Geurts A, Sidjanin DJ. Targeted disruption of Tbc1d20 with zinc-finger nucleases causes cataracts and testicular abnormalities in mice. BMC Genetics 2014;15:135.

[25] Salabi F, Nazari M, Chen Q, Nimal J, Tong J, Cao WG. Myostatin knockout using zinc-finger nucleases promotes proliferation of ovine primary satellite cells in vitro. Journal of Biotechnology 2014;192PA:268-80.

[26] Sampson KE, Brinker A, Pratt J, Venkatraman N, Xiao Y, Blasberg J, Steiner T, Bourner M, Thompson DC. Zinc finger nuclease-mediated gene knockout results in loss of transport activity for P-glycoprotein, BCRP, and MRP2 in Caco-2 cells. Drug metabolism and disposition: the biological fate of chemicals 2015;43:199-207.

[27] Ding W, Hu Z, Zhu D, Jiang X, Yu L, Wang X, Zhang C, Wang L, Ji T, Li K, He D, Xia X, Liu D, Zhou J, Ma D, Wang H. Zinc finger nucleases targeting the human papillomavirus E7 oncogene induce E7 disruption and a transformed phenotype in HPV16/18-positive cervical cancer cells. Clinical cancer research: an official journal of the American Association for Cancer Research 2014;20:6495-503.

[28] Yi G, et al. CCR5 gene editing of resting CD4(+) T cells by transient ZFN expression from HIV envelope pseudotyped nonintegrating lentivirus confers HIV-1 resistance in humanized mice. Molecular Therapy Nucleic Acids 2014;3:e198.

[29] Liu X, et al. Generation of mastitis resistance in cows by targeting human lysozyme gene to beta-casein locus using zinc-finger nucleases. Proceedings Biological Sciences / The Royal Society 2014;281:20133368.

[30] Lombardo A, et al. Site-specific integration and tailoring of cassette design for sustainable gene transfer. Nature Methods 2011;8:861-9.

[31] Merling RK, Sweeney CL, Chu J, Bodansky A, Choi U, Priel DL, Kuhns DB, Wang H, Vasilevsky S, De Ravin SS, Winkler T, Dunbar CE, Zou J, Zarember KA, Gallin JI, Holland SM, Malech HL. An AAVS1-targeted minigene platform for correction of iPSCs from all five types of chronic granulomatous disease. Molecular therapy: the journal of the American Society of Gene Therapy 2015;23:147-57.

[32] Zhang W, et al. Targeted genome correction by a single adenoviral vector simultaneously carrying an inducible zinc finger nuclease and a donor template. Journal of Biotechnology 2014;188C:1-6.

[33] Zou J, Mali P, Huang X, Dowey SN, Cheng L. Site-specific gene correction of a point mutation in human iPS cells derived from an adult patient with sickle cell disease. Blood 2011;118:4599-608.

[34] Sebastiano V, et al. In situ genetic correction of the sickle cell anemia mutation in human induced pluripotent stem cells using engineered zinc finger nucleases. Stem Cells 2011;29:1717-26.

[35] Bogdanove AJ, Voytas DF. TAL effectors: Customizable proteins for DNA targeting. Science 2011;333:1843-6.

[36] Kay S, Hahn S, Marois E, Hause G, Bonas U. A bacterial effector acts as a plant transcription factor and induces a cell size regulator. Science 2007;318:648-51. 
[37] Bogdanove AJ, Schornack S, Lahaye T. TAL effectors: Finding plant genes for disease and defense. Current Opinion in Plant Biology 2010;13:394-401.

[38] Cermak T, et al. Efficient design and assembly of custom TALEN and other TAL effector-based constructs for DNA targeting. Nucleic Acids Research 2011;39:e82.

[39] Moscou MJ, Bogdanove AJ. A simple cipher governs DNA recognition by TAL effectors. Science 2009;326:1501.

[40] Miller JC, et al. A TALE nuclease architecture for efficient genome editing. Nature Biotechnology 2011;29:143-8.

[41] Zhang F, Cong L, Lodato S, Kosuri S, Church GM, Arlotta P. Efficient construction of sequence-specific TAL effectors for modulating mammalian transcription. Nature Biotechnology 2011;29:149-53.

[42] Li T, et al. TAL nucleases (TALNs): Hybrid proteins composed of TAL effectors and FokI DNA-cleavage domain. Nucleic Acids Research 2011;39:359-72.

[43] Zhang Z, Wu E, Qian Z, Wu WS. A multicolor panel of TALE-KRAB based transcriptional repressor vectors enabling knockdown of multiple gene targets. Scientific Reports 2014;4:7338.

[44] Elbaz I, Lerer-Goldshtein T, Okamoto H, Appelbaum L. Reduced synaptic density and deficient locomotor response in neuronal activity-regulated pentraxin 2a mutant zebrafish. FASEB Journal: Official Publication of the Federation of American Societies for Experimental Biology 2014.

[45] Choi J, Suzuki KI, Sakuma T, Shewade L, Yamamoto T, Buchholz DR. Unliganded thyroid hormone receptor alpha regulates developmental timing via gene repression as revealed by gene disruption in Xenopus tropicalis. Endocrinology 2014; en20141554.

[46] Kabir S, Hockemeyer D, de Lange T. TALEN gene knockouts reveal no requirement for the conserved human shelterin protein Rap1 in telomere protection and length regulation. Cell Reports 2014;9:1273-80.

[47] Lugassy J, et al. Modulation of TCR responsiveness by the Grb2-family adaptor, Gads. Cellular Signalling 2014;27:125-34.

[48] Tatsumi Y, Takeda M, Matsuda M, Suzuki T, Yokoi H. TALEN-mediated mutagenesis in zebrafish reveals a role for r-spondin 2 in fin ray and vertebral development. FEBS Letters 2014;588:4543-50.

[49] Liu C, Xiao L, Li F, Zhang H, Li Q, Liu H, Fu S, Li C, Zhang X, Wang J, Staunstrup NH, Li Y, Yang H. Generation of outbred Ace2 knockout mice by RNA transfection of TALENs displaying colitis reminiscent pathophysiology and inflammation. Transgenic research (2014).

[50] Zhang Z, Zhu B, Ge W. Genetic analysis of zebrafish gonadotropin (FSH and LH) functions by TALEN-mediated gene disruption. Molecular Endocrinology 2015;29:76-98.

[51] Wen L, Fu L, Guo X, Chen Y, Shi YB. Histone methyltransferase Dot1L plays a role in postembryonic development in Xenopus tropicalis. FASEB Journal: Official Publication of the Federation of American Societies for Experimental Biology 2015;29:385-93.

[52] Chen B, et al. Disruption of microRNA-21 by TALEN leads to diminished cell transformation and increased expression of cell-environment interaction genes. Cancer Letters 2015;356:506-16.

[53] Krentz NA, Nian C, Lynn FC. TALEN/CRISPR-mediated eGFP knock-in add-on at the OCT4 locus does not impact differentiation of human embryonic stem Cells towards endoderm. PloS One 2014;9:e114275.

[54] Li HL, Fujimoto N, Sasakawa N, Shirai S, Ohkame T, Sakuma T, Tanaka M, Amano N, Watanabe A, Sakurai H, Yamamoto T, Yamanaka S, Hotta A. Precise correction of the dystrophin gene in duchenne muscular dystrophy patient induced pluripotent stem cells by TALEN and CRISPR-Cas9. Stem cell reports 2015;4:143-54.

[55] Ousterout DG, et al. Reading frame correction by targeted genome editing restores dystrophin expression in cells from Duchenne muscular dystrophy patients. Molecular Therapy: The Journal of the American Society of Gene Therapy 2013;21:1718-26.

[56] Ramalingam S, Annaluru N, Kandavelou K, Chandrasegaran S. TALEN-Mediated generation and genetic correction of disease-specific human induced pluripotent stem cells. Current Gene Therapy 2014;14:461-72.

[57] Suzuki K, et al. Targeted gene correction minimally impacts whole-genome mutational load in human-disease-specific induced pluripotent stem cell clones. Cell Stem Cell 2014;15:31-6.

[58] Ma N, et al. Transcription activator-like effector nuclease (TALEN)-mediated gene correction in integration-free betathalassemia induced pluripotent stem cells. The Journal of Biological Chemistry 2013;288:34671-9.

[59] Sun N, Zhao H. Seamless correction of the sickle cell disease mutation of the HBB gene in human induced pluripotent stem cells using TALENs. Biotechnology and Bioengineering 2014;111:1048-53.

[60] Low BE, Krebs MP, Joung JK, Tsai SQ, Nishina PM, Wiles MV. Correction of the Crb1rd8 allele and retinal phenotype in C57BL/6N mice via TALEN-mediated homology-directed repair. Investigative Ophthalmology \& Visual Science 2014;55:387-95. 
[61] Dupuy A, et al. Targeted gene therapy of xeroderma pigmentosum cells using meganuclease and TALEN. PloS One 2013;8:e78678.

[62] Ishino Y, Shinagawa H, Makino K, Amemura M, Nakata A. Nucleotide sequence of the iap gene, responsible for alkaline phosphatase isozyme conversion in Escherichia coli, and identification of the gene product. Journal of Bacteriology 1987;169:5429-33.

[63] Jansen R, Embden JD, Gaastra W, Schouls LM. Identification of genes that are associated with DNA repeats in prokaryotes. Molecular Microbiology 2002;43:1565-75.

[64] Shah SA, Erdmann S, Mojica FJ, Garrett RA. Protospacer recognition motifs: Mixed identities and functional diversity. RNA Biology 2013;10:891-9.

[65] Mojica FJ, Diez-Villasenor C, Garcia-Martinez J, Almendros C. Short motif sequences determine the targets of the prokaryotic CRISPR defence system. Microbiology 2009;155:733-40.

[66] Pougach K, et al. Transcription, processing and function of CRISPR cassettes in Escherichia coli. Molecular Microbiology 2010;77:1367-79.

[67] Wiedenheft B, Sternberg SH, Doudna JA. RNA-guided genetic silencing systems in bacteria and archaea. Nature 2012;482:331-8.

[68] Deltcheva E, et al. CRISPR RNA maturation by trans-encoded small RNA and host factor RNase III. Nature 2011;471:602-7.

[69] Gesner EM, Schellenberg MJ, Garside EL, George MM, Macmillan AM. Recognition and maturation of effector RNAs in a CRISPR interference pathway. Nature Structural \& Molecular Biology 2011;18:688-92.

[70] Garneau JE, et al. The CRISPR/Cas bacterial immune system cleaves bacteriophage and plasmid DNA. Nature 2010;468:67-71.

[71] Makarova KS, et al. Evolution and classification of the CRISPR-Cas systems. Nature Reviews Microbiology 2011;9:467-77.

[72] Sternberg SH, Redding S, Jinek M, Greene EC, Doudna JA. DNA interrogation by the CRISPR RNA-guided endonuclease Cas9. Nature 2014;507:62-7.

[73] Mehrabian M, et al. CRISPR-Cas9-based knockout of the prion protein and its effect on the proteome. PloS One 2014;9:e114594.

[74] Cho SW, Kim S, Kim JM, Kim JS. Targeted genome engineering in human cells with the Cas9 RNA-guided endonuclease. Nature Biotechnology 2013;31:230-2.

[75] Cong L, et al. Multiplex genome engineering using CRISPR/Cas systems. Science 2013;339:819-23.

[76] Mali P, et al. RNA-guided human genome engineering via Cas9. Science 2013;339:823-6.

[77] Wu Y, Zhou H, Fan X, Zhang Y, Zhang M, Wang Y, Xie Z, Bai M, Yin Q, Liang D, Tang W, Liao J, Zhou C, Liu W, Zhu P, Guo H, Pan H, Wu C, Shi H, Wu L, Tang F, Li J. Correction of a genetic disease by CRISPR-Cas9-mediated gene editing in mouse spermatogonial stem cells. Cell research 2015;25:67-79.

[78] Long C, McAnally JR, Shelton JM, Mireault AA, Bassel-Duby R, Olson EN. Prevention of muscular dystrophy in mice by CRISPR/Cas9-mediated editing of germline DNA. Science 2014;345:1184-8.

[79] Chen L, et al. Advances in genome editing technology and its promising application in evolutionary and ecological studies. Giga Science 2014;3:24.

[80] Hsu PD, Lander ES, Zhang F. Development and applications of CRISPR-Cas9 for genome engineering. Cell 2014;157:1262-78.

[81] Konermann S, Brigham MD, Trevino AE, Joung J, Abudayyeh OO, Barcena C, Hsu PD, Habib N, Gootenberg JS, Nishimasu H, Nureki O, Zhang F. Genome-scale transcriptional activation by an engineered CRISPR-Cas9 complex. Nature 2015;517:583-8.

[82] Urnov FD, et al. Highly efficient endogenous human gene correction using designed zinc-finger nucleases. Nature 2005;435:646-51.

[83] Li H, et al. In vivo genome editing restores haemostasis in a mouse model of haemophilia. Nature 2011;475:217-21. 